\title{
Masses and phase structure in the Ginzburg-Landau model
}

\author{
K. Kajantie* \\ Theory Division, CERN, CH-1211 Geneva 23, Switzerland \\ and Department of Physics, P.O. Box 9, 00014 University of Helsinki, Finland \\ M. Karjalainen ${ }^{\dagger}$ \\ Department of Physics, P.O. Box 9, 00014 University of Helsinki, Finland
}

M. Laine

Institut für Theoretische Physik, Philosophenweg 16, D-69120 Heidelberg, Germany

J. Peisa ${ }^{\S}$

Department of Mathematical Sciences, University of Liverpool, Liverpool L69 3BX, United Kingdom

(Received 1 August 1997)

\begin{abstract}
We study numerically the phase structure of the Ginzburg-Landau model, with particular emphasis on mass measurements. There is no local gauge-invariant order parameter, but we find that there is a phase transition characterized by a vanishing photon mass. For type-I superconductors the transition is of first order. For type-II, a first-order transition is excluded by susceptibility analysis, but the photon correlation length is compatible with second-order critical behavior with $\nu \sim \frac{1}{2}$. The scalar mass, in contrast, does not show clear critical behavior in the type-II regime for $V \rightarrow \infty$, contrary to the conventional picture. [S0163-1829(98)04205-2]
\end{abstract}

\section{INTRODUCTION}

One of the most interesting phase transitions known is that some materials become superconductive at low temperatures. While the microscopic dynamics behind the phenomenon is complicated, there exists a simple effective theory for describing this transition. The effective theory is just the Ginzburg-Landau (GL) model, or a three-dimensional U(1) + complex scalar gauge theory. The modulus squared of the scalar field represents the density of superconductive electrons. The GL model might have other applications as well, such as the nematic-to-smectic-A transition in liquid crystals. ${ }^{1}$

There exists an extensive literature on the phase diagram of the GL model. ${ }^{2-7}$ Defining the standard GL parameter as $x=m_{H}^{2} / 2 m_{W}^{2}$ (where $m_{H}$ is the inverse scalar correlation length, or coherence length, and $m_{W}$ is the inverse vector correlation length, or penetration depth), the conventional picture is that the transition is of first order for small $x$ (type-I superconductors), gets weaker with increasing $x$, has a tricritical point at some $x_{c}$, and remains of second order for $x>x_{c}$ (type-II superconductors). ${ }^{8}$ This picture is based on perturbative, renormalization-group and lattice studies, often in a dual theory. These studies have nevertheless not been conclusive and there have been arguments concerning, for instance, the universality class of the provisional secondorder transition in the type-II regime $(x>1 / 2) .^{7}$

The purpose of this paper is to study the phase diagram numerically. We improve significantly upon earlier numerical results, ${ }^{3,4}$ by having a much finer lattice (smaller lattice constant $a$ in physical units) and by measuring the different correlation lengths. While the infinite volume and continuum extrapolations are numerically demanding and thus the con- clusions based on a series of finite lattices can never be quite complete, we nevertheless find indications of quite an unexpected pattern in the type-II regime. We also point out directions for future investigations of this issue.

The paper is organized as follows. In Sec. II we specify the model studied, in Sec. III we discuss how it is discretized, and in Sec. IV how the simulations are organized and what our results are. Section $\mathrm{V}$ is a discussion.

\section{THE MODEL}

Let us first define the model unambiguously. It is a locally gauge-invariant three-dimensional continuum $\mathrm{U}(1)+$ complex scalar field theory defined by the functional integral

$$
\begin{gathered}
Z=\int \mathcal{D} A_{i} \mathcal{D} \phi \exp \left[-S\left(A_{i}, \phi\right)\right], \\
S=\int d^{3} x\left[\frac{1}{4}\left(\partial_{i} A_{j}-\partial_{j} A_{i}\right)^{2}+\left|\left(\partial_{i}+i e_{3} A_{i}\right) \phi\right|^{2}\right. \\
\left.+m_{3}^{2} \phi^{*} \phi+\lambda_{3}\left(\phi^{*} \phi\right)^{2}\right] .
\end{gathered}
$$

The couplings $e_{3}^{2}, \lambda_{3}$ have the dimension of mass (in units $\hbar=c=1)$ and, factoring out one scale $\left(e_{3}^{2}\right)$, the free-energy density $f$ of the model depends on the two dimensionless ratios

$$
y \equiv \frac{m_{3}^{2}}{e_{3}^{4}}, \quad x \equiv \frac{\lambda_{3}}{e_{3}^{2}},
$$

so that 


$$
Z=\exp \left[-V e_{3}^{6} f(y, x)\right] .
$$

The phase diagram of the theory can thus be drawn in the $(x, y)$ plane.

Since the theory in Eq. (2) is a continuum field theory, one has to consider ultraviolet renormalization. There is a linear one-loop and logarithmic two-loop divergence ${ }^{11}$ for the mass parameter $m_{3}^{2}$. In the $\overline{\mathrm{MS}}$ dimensional regularization scheme in $3-2 \epsilon$ dimensions, the renormalized mass parameter becomes ${ }^{12}$

$$
m_{3}^{2}(\mu)=\frac{-4 e_{3}^{4}+8 \lambda_{3} e_{3}^{2}-8 \lambda_{3}^{2}}{16 \pi^{2}} \ln \frac{\Lambda_{m}}{\mu},
$$

where $\Lambda_{m}$ specifies the theory. To be more precise, we thus define $y \equiv m_{3}^{2}\left(e_{3}^{2}\right) / e_{3}^{4}$, which specifies the continuum theory at the full quantum level equally well as $\Lambda_{m}$.

The physical values of $x$ and $y$ depend on the microscopic theory behind the effective theory in Eq. (2). For reference, for usual superconductors in the notation of Ref. 5,

$$
y=\frac{1}{r q^{4}}\left(\frac{T}{T_{c}}-1\right), \quad x=\frac{g}{(r q)^{2}} \sim \frac{0.01}{r^{2}},
$$

with $g \sim 10^{-6}, q \sim 0.01, r \leqslant 1$. For high- $T_{c}$ superconductors $x$ can be $\gg 1$. At present we consider the theory (2) as such and questions of validity, like the need to include $\left(\phi^{*} \phi\right)^{3}$ terms, do not enter.

The phase diagram of the GL model on the $y, x>0$ plane (see Fig. 1) contains a curve $y=y_{c}(x)$ along which the system has a first-order transition for small $x$. To one vector loop this curve is simply given by $y_{c}=1 /\left(18 \pi^{2} x\right)$. We shall

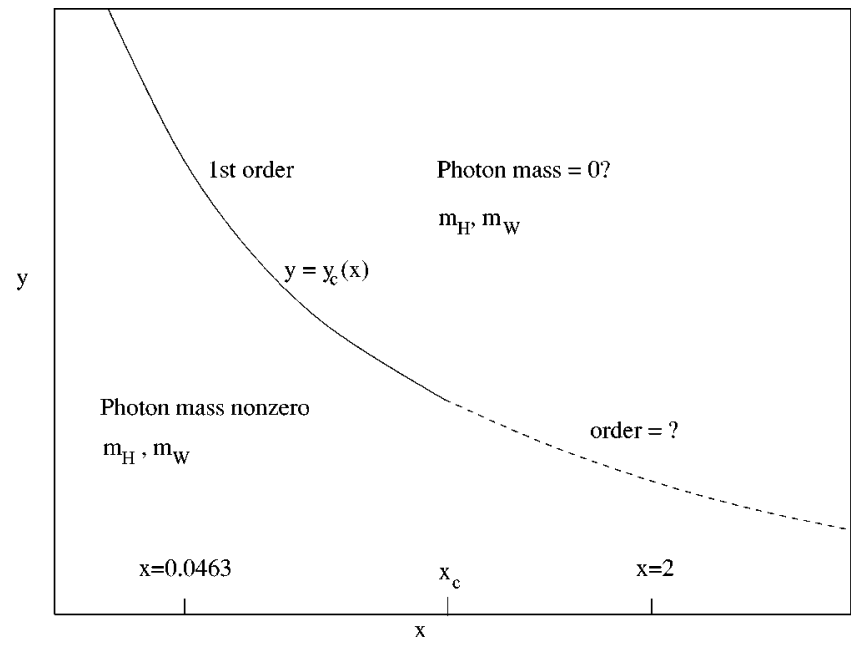

FIG. 1. The qualitative phase diagram of the GL theory.

call the region $y>y_{c}(x)$ the normal and the region $y$ $<y_{c}(x)$ the superconducting (SC) phase. At small $x$ the reliability of perturbation theory has been verified numerically. ${ }^{13}$ However, perturbation theory gets worse at large $x$ and the issue now is what happens then.

\section{DISCRETIZATION}

To latticize ( $a=$ lattice spacing) the theory with the fixed continuum variables $x, y$, we introduce the link field $U_{i}(\mathbf{x})$ $=\exp \left[\operatorname{iae}_{3} A_{i}(\mathbf{x})\right] \equiv \exp \left[i \alpha_{i}(\mathbf{x})\right]$. Relating the counterterms in the $\overline{\mathrm{MS}}$ and lattice regularization schemes, ${ }^{14}$ the lattice action becomes ${ }^{15}$

$$
\begin{aligned}
S= & \beta_{G} \sum_{\mathbf{x}, i<j}\left[1-\cos \hat{F}_{i j}(\mathbf{x})\right]-\beta_{H} \sum_{\mathbf{x}, i} \operatorname{Re} \phi^{*}(\mathbf{x}) U_{i}(\mathbf{x}) \phi(\mathbf{x}+\hat{i})+\frac{\beta_{H}}{2} \sum_{\mathbf{x}} \phi^{*}(\mathbf{x}) \phi(\mathbf{x})\left[6+\frac{y}{\beta_{G}^{2}}-\frac{3.1759115(1+2 x)}{2 \pi \beta_{G}}\right. \\
& \left.-\frac{\left(-4+8 x-8 x^{2}\right)\left(\ln 6 \beta_{G}+0.09\right)+25.5+4.6 x}{16 \pi^{2} \beta_{G}^{2}}\right]+\frac{x \beta_{H}^{2}}{4 \beta_{G}} \sum_{\mathbf{x}}\left[\phi^{*}(\mathbf{x}) \phi(\mathbf{x})\right]^{2},
\end{aligned}
$$

where $\beta_{H}$ is so far arbitrary, $\beta_{G}=1 / e_{3}^{2} a$, and

$$
\hat{F}_{i j}(\mathbf{x})=\alpha_{i}(\mathbf{x})+\alpha_{j}(\mathbf{x}+\hat{i})-\alpha_{i}(\mathbf{x}+\hat{j})-\alpha_{j}(\mathbf{x}) .
$$

We have scaled the continuum scalar field to a dimensionless lattice field by $\phi^{*} \phi \rightarrow \beta_{H} \phi^{*} \phi / 2 a$, but further rescalings (by a specific choice of $\beta_{H}$ ) are possible; we thus scale the coefficient of $\phi^{*} \phi$ to be +1 . For a given continuum theory $\left(e_{3}^{2}, y, x\right)$, Eqs. (7),(8) specify up to terms of order $e_{3}^{2} a$ the corresponding lattice action. It should be noted that the complicated counterterm expression in the square brackets in Eq. (7) only affects the value of $y_{c}$ for given $x$ [the counterterm guarantees that the limit $y_{c}(a \rightarrow 0)$ exists], but not the qualitative structure of the phase diagram.

One of the most essential points of the present lattice simulations is the extrapolation to the continuum limit: first the infinite volume $V \rightarrow \infty$ at fixed lattice spacing $a$, then $a \rightarrow 0$. To estimate the required sizes of $V=(N a)^{3}$ and $a$, consider a system with a typical correlation length $\xi$. Then one has to satisfy (on a periodic lattice) $a \ll \xi \ll N a / 2$ or, in physical units,

$$
e_{3}^{2} a=\frac{1}{\beta_{G}} \ll e_{3}^{2} \xi \ll \frac{N}{2 \beta_{G}} .
$$

We observe that $e_{3}^{2} \xi \sim 1$ and take $\beta_{G}=4,6$ so that $1 / \beta_{G}$ $\ll e_{3}^{2} \xi$; and $N=32, \ldots, 64$ so that $N /\left(2 \beta_{G}\right) \gg e_{3}^{2} \xi$. Note that Ref. 4 had $\beta_{G}=0.2, N \leqslant 15$, so that the lattice spacing $a$ was larger than the typical correlation lengths.

Apart from the UV-cutoff effects discussed, there is another effect related to a finite $a$. Indeed, we use a compact formulation for the $\mathrm{U}(1)$ gauge field, which changes the topology of the theory and implies that the photon becomes massive. ${ }^{16}$ However, a semiclassical computation for this Polyakov mass gives ${ }^{17}$ 


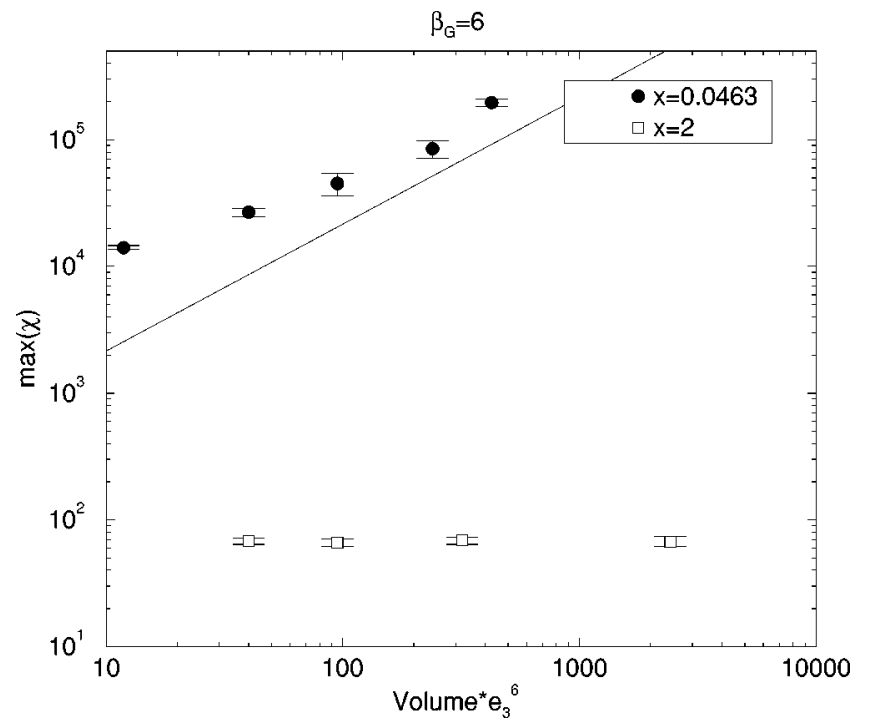

FIG. 2. The maximum of the susceptibility $\chi$ as a function of volume. The straight line is $\sim V$.

$$
\frac{m_{\gamma}^{P}}{e_{3}^{2}}=\pi\left(2 \beta_{G}\right)^{3 / 2} \exp \left[-\frac{3.176 \pi}{4} \beta_{G}\right] .
$$

Thus for $\beta_{G} \geqslant 4$ this photon mass (as well as the monopole density from which it originates) should be negligible $\left(m_{\gamma}^{P} / e_{3}^{2}<0.01\right)$ and our results the same as in the noncompact formulation within statistical errors.

\section{SIMULATIONS AND RESULTS}

For the simulations we choose two values of $x, x$ $=0.0463$ and $x=2$, corresponding to strongly type-I and type-II superconductors. We then measure averages of local or bilocal gauge-invariant quantities and locate the critical curve $y=y_{c}(x)$ on which the system changes its properties. Note that there is no local gauge-invariant order parameter which would vanish in either of the phases. However, we shall find that the photon mass, measured from a correlator, vanishes in one of the phases within errors.

The phase transition is located by finding the maximum in $y$ of the susceptibility $\chi$ defined by

$$
\chi=e_{3}^{2} V\left\langle\left(\phi^{* \phi}-\left\langle\phi^{* \phi}\right\rangle\right)^{2}\right\rangle,
$$

where $\overline{\phi^{*} \phi}$ is the volume average, $\overline{\phi^{*} \phi}=V^{-1} \int d^{3} x \phi^{*} \phi$, and by studying its large- $V$ behavior. ${ }^{18}$ If there is a first-order transition, the distribution of $\overline{\phi^{*} \phi}$ precisely at $y_{c}(x)$ has two peaks which remain at fixed distance and get narrower when $V \rightarrow \infty$. Then the maximum of $\chi$ grows as the volume $V$. In a second-order transition the expected behavior is $\sim V^{\kappa}, \kappa$ being a critical exponent. If $\chi \sim V^{0}$, then either $\kappa \leqslant 0$, or the transition is of higher than second order or absent.

The susceptibility maximum is plotted in Fig. 2. One sees a very clear difference between $x=0.0463$ and $x=2$. The behavior of the system at $x=0.0463$ at the largest volumes indicates a linear first-order behavior. At $x=2$, in contrast, the transition is not of first order. If the transition is of second order the critical exponent $\kappa$ is close to zero, as noticed already in Refs. 3,4. However, a still higher-order transition (or a smooth crossover) cannot be excluded based on these measurements. We thus turn to masses.

For the mass measurements we use the scalar operator $\phi^{*} \phi$ and the two vector operators $\phi^{*} D_{i} \phi$ and $\epsilon_{i j k} F_{j k}$. On the lattice these are

$$
\begin{gathered}
O(\mathbf{x})=\phi^{*}(\mathbf{x}) \phi(\mathbf{x}) \\
O_{i}(\mathbf{x})=\operatorname{Im} \phi^{*}(\mathbf{x}) U_{i}(\mathbf{x}) \phi(\mathbf{x}+\hat{i}), \\
\widetilde{O}_{i}(\mathbf{x})=\epsilon_{i j k} \sin \hat{F}_{j k}(\mathbf{x}) .
\end{gathered}
$$

The correlation masses are measured (from a lattice of size $N_{x}^{2} N_{z}$ ) by first summing over planes, possibly with momentum $p=2 \pi /\left(a N_{x}\right)$,

$$
O(z ; p)=\sum_{x, y} e^{i p x} O(x, y, z),
$$

and then studying the large- $t$ behavior of

$$
G(t ; p)=\frac{1}{N_{x}^{2} N_{z}} \sum_{z}\left\langle O(z ; p) O^{*}(z+t ; p)\right\rangle .
$$

The momentum is needed for the correlator of $\widetilde{O}_{3}$, used to measure the photon mass ${ }^{19}$ (it can also be used for the very light scalar mass, to get a better signal in a finite volume): without the factor $e^{i p x}$ in Eq. (15), the plane average for $\widetilde{O_{3}}$ would simply vanish. In perturbation theory, one finds for the asymptotic behavior of this correlator in the continuum limit,

$$
G_{3}(t)=\frac{A_{\gamma}}{\beta_{G}} \frac{a p^{2}}{2 E} e^{-E t}, \quad E^{2}=p^{2}+m_{\gamma}^{2},
$$

where $m_{\gamma}=0$ in the normal phase. At the one-loop level for $y>0$,

$$
A_{\gamma}=1-\frac{1}{24 \pi \sqrt{y}} \text {. }
$$

Very close to the critical point $y \sim 0$ the expansion for $A_{\gamma}$ thus breaks down, and the form of the correlation function is determined by an anomalous dimension. For our datapoints, the functional form in Eq. (17) fits the data well and the energy $E$ is measured from the exponential falloff. The mass is then obtained from $m=\sqrt{E^{2}-p^{2}}$.

To improve the projection to the low-lying mass states, it is indispensable ${ }^{20}$ to use blocking techniques ${ }^{22}$ to define extended operators, and to make a mixing analysis ${ }^{22}$ between operators at different blocking levels, to search for the linear combination giving the best signal. We find the best results with blocking level 3 for the scalar mass. The mixing analysis allows us to get a good signal already at a relatively small $t, t \sim(2-5) a$. We denote by $m_{\gamma}$ the lowest-lying vector state, and by $m_{W}$ the first excitation (where it can be determined).

The masses $(=1 / \xi)$ near the transition are shown in Fig. 3 . For type-I superconductors $(x=0.0463)$ one observes in the SC phase one scalar with a rather small mass $m_{H}$. The vector operators couple dominantly to a single state of a larger mass $m_{W}=m_{\gamma}$. These are the standard (inverse) co- 

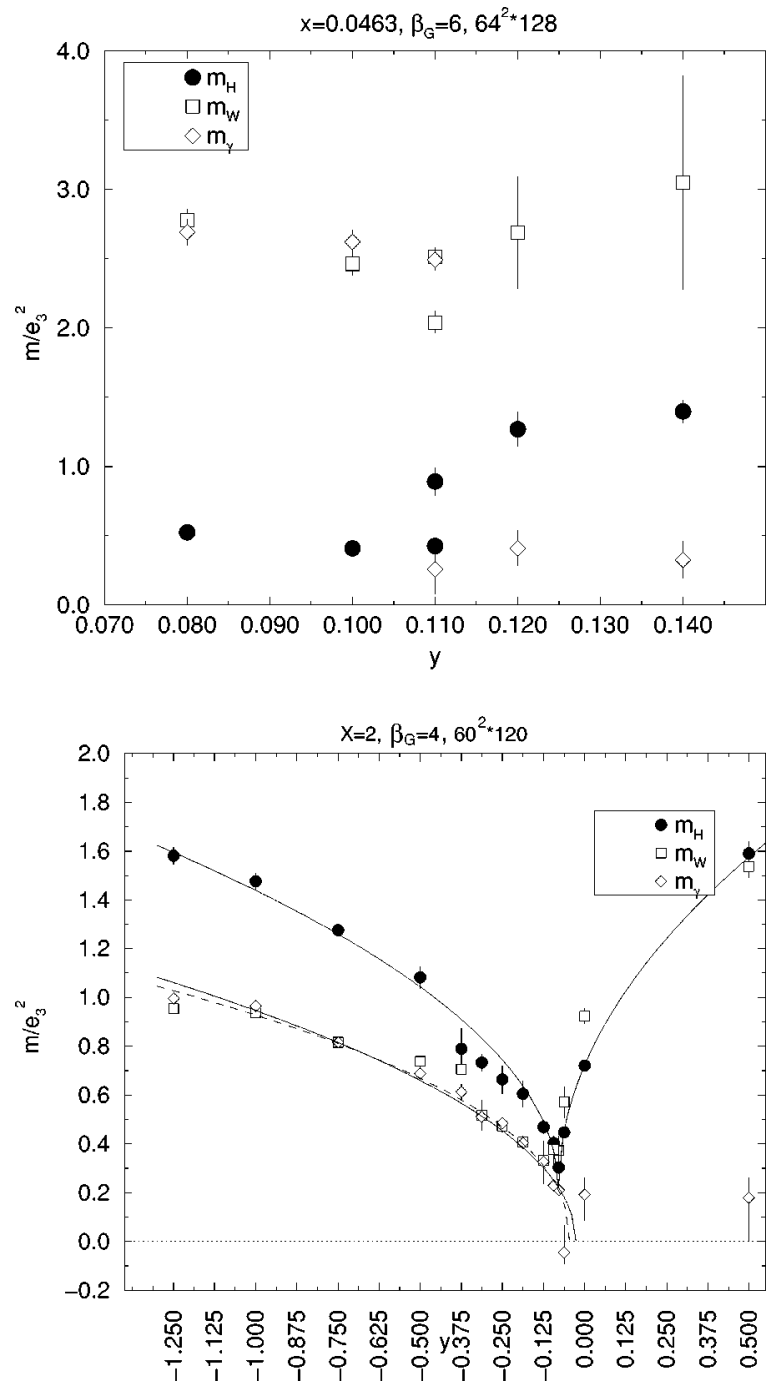

FIG. 3. The masses at $x=0.0463$ (top) and $x=2$ (bottom). Note the discontinuous and continuous behavior of $m_{\gamma}$ in the top and bottom figures, respectively. The values of $m_{\gamma}$ in the top figure for $y=0.12,0.14$ are $2 \ldots 3$ standard deviations from zero, but all or most of this deviation is expected to be due to the absence of a mixing analysis at these data points. The curves in the bottom figure represent fits $\sim A\left|y-y_{c}\right|^{\nu}$. The dashed curve for $m_{\gamma}$ is with a free exponent $\nu=0.44(2)$ over the whole $y$ range whereas for the solid curves, $\nu$ has been fixed to $\nu=\frac{1}{2}$.

herence length and penetration depth. Close to the transition point one can observe both phases simultaneously, and the masses are discontinuous. Above the transition the scalar excitation is there with a larger mass, but the vector operators couple to two quite different states. There is a $\phi^{*} \phi$ bound state of large mass $m_{W}$, seen dominantly by the operator $O_{i}$, while the operator $\widetilde{O_{3}}$ sees the photon of mass $m_{\gamma}$ which is consistent with zero within $2 \sigma$. The picture here is the standard one of a first-order transition with $m_{\gamma}$ as an effective order parameter.

For type-II superconductors $(x=2)$ a first-order transition was excluded by the susceptibility analysis: no two-peak structure exists. However, Fig. 3 shows that there is some transition since in the normal phase $m_{\gamma}=0$ within errors. The critical region is shown in a magnified form in Fig. 4, and one can see that $m_{\gamma}$ could go to zero continuously (on

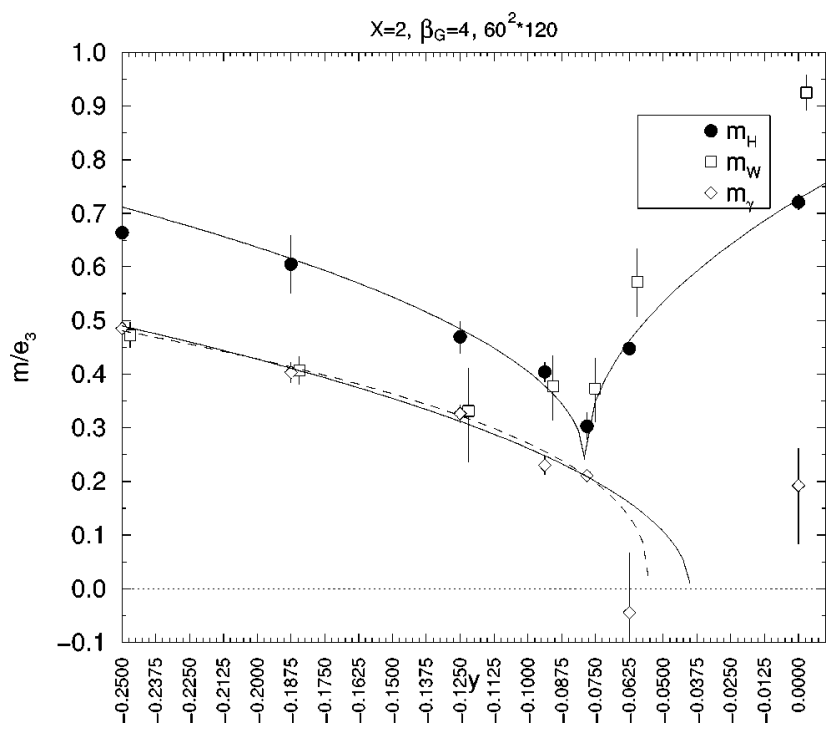

FIG. 4. A magnification of the region around the critical point for $x=2$. The dashed curve is a fit with a free exponent over the range $y=-0.25 \ldots 0.0$, and gives $\nu=0.39(17)$. The solid curves are with $\nu=\frac{1}{2}$, and with a constant for $m_{H}$ (see Fig. 5).

the other hand, it should be noted that a discontinuity in $m_{\gamma}$ cannot be excluded either). The dashed curve in Fig. 3 shows the fit $m_{\gamma} / e_{3}^{2}=A\left(y_{c}-y\right)^{\nu}$ over the whole $y$ range, where $A$ $=0.95(2), y_{c}=-0.046(6), \nu=0.44(2)$. For the lower solid curve $\nu$ has been fixed to $\nu=\frac{1}{2}$. In Fig. 4 (for a smaller $y$ range), $\nu=0.39(17)$. The behavior of $m_{\gamma}$ is thus consistent with a mean-field exponent.

As to $m_{W}$, it is seen that it deviates from $m_{\gamma}$ already below the critical point, unlike in the first-order case shown in the top panel of Fig. 3. This might indicate that the transition line has split into several transitions in the type-II regime. However, $m_{W}$ is an excited state and thus there are some systematic errors in its determination which may be larger than the statistical errors shown in the figure.

Consider then the scalar mass $m_{H}$. First, note that the data in Fig. 4 indicated that its minimum is at a point different from where $m_{\gamma}$ goes to zero, assuming the $m_{\gamma}$ behaves continuously. The other possibility is that $m_{\gamma}$ jumps to zero at the point where $m_{H}$ is at a minimum. Second, $m_{H}$ dips steeply in Fig. 3, but does not go to zero as can be seen in Fig. 4. On a finite (periodic) lattice with spatial extension $L=N a$ one cannot expect to see scalar mass values smaller than $\sim 2 / L$. However, according to Fig. 5 the scalar mass is larger than this value and does not show the corresponding volume dependence. In fact, $m_{H}$ is volume independent within error bars at the minimum.

The question remaining then is whether the scalar mass depends on the lattice spacing $a$. This seems unlikely, since the lattice spacing we used is much smaller than the correlation length, $e_{3}^{2} a=1 / \beta_{G}=1 / 4 \ll e_{3}^{2} \xi_{H}=e_{3}^{2} / m_{H} \approx 2$, so that one does not expect large effects from removing the UV cutoff. Indeed, we have made simulations with $\beta_{G}=3$ and $\beta_{G}=6$ at a few points around the minimum, and we do not find any appreciable lattice spacing dependence. It should be pointed out, though, that for $\beta_{G}=6$ one should go to $\sim 50 \%$ larger lattice sizes $N$ to get physical volumes comparable with those for $\beta_{G}=4$, according to Eq. (9). 


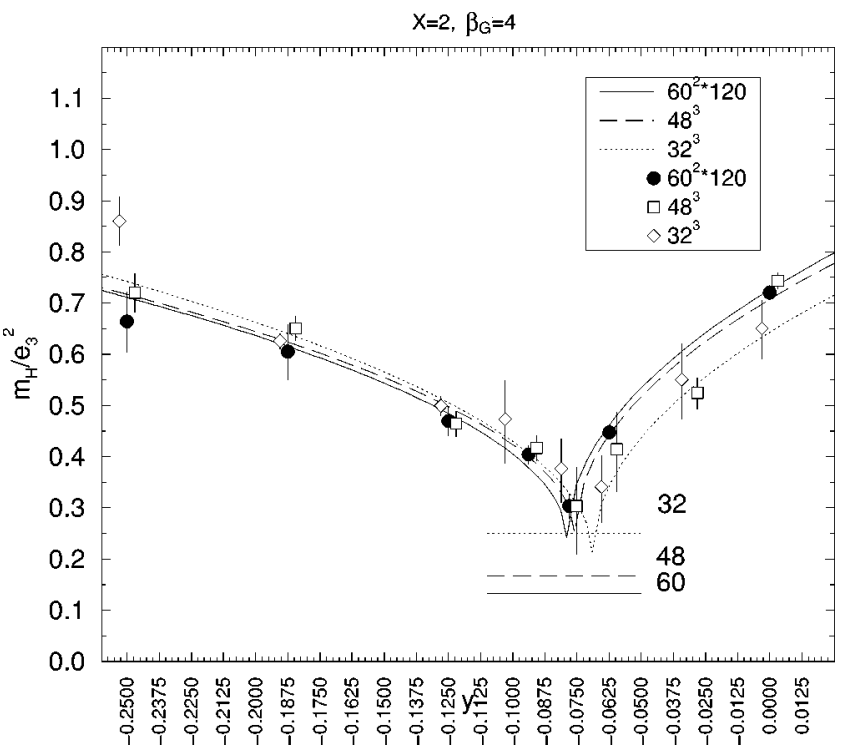

FIG. 5. The volume dependence of the scalar mass. The results are consistent within error bars at the minimum. The expected critical scaling behavior is shown with the horizontal lines indicating the value of $2 / L$. No such scaling is observed. The fits are of the form $m_{H} / e_{3}^{2} \sim\left[A^{\prime}\left(y_{c}-y\right)^{1 / 2}+B\right]\left(y<y_{c}\right),\left[A\left(y-y_{c}\right)^{1 / 2}+B\right](y$ $>y_{c}$ ), with $A^{\prime} \sim 1.1, A \sim 1.7$.

Note also that as long as $a \neq 0$ there is in a strict sense no phase transition due to the Polyakov mass. However, the Polyakov mass in Eq. (10) is very small for $\beta_{G}=4$, so that its finite value should have no effect.

\section{DISCUSSION}

We have seen that in the type-II regime, the data are consistent with a second-order phase transition driven by a diverging photon correlation length. In contrast, the scalar mass shows quite unexpected behavior. It appears that $m_{H}$ has a minimum away from where the photon correlation length diverges (assuming that $m_{\gamma}$ behaves continuously), and that $m_{H}$ does not show critical scaling towards zero when the volume increases. One can also envisage a scenario in which there is only one critical point at which $m_{\gamma}$ drops to zero, $m_{H}$ has its finite minimum and the excited vector state $m_{W}$ decouples from the photon. If this pattern remains there for larger volumes and smaller lattice spacings, then one has to modify the standard picture of the superconducting phase transition in the type-II regime.
As to the critical exponents of the transition, we have measured the finite-size scaling susceptibility exponent defined after Eq. (11) to be consistent with zero, and the photon correlation length exponent in the SC phase to be consistent with $\frac{1}{2}$. We have not measured the anomalous dimension $\eta_{A}$ of the photon correlation length, $\left\langle\widetilde{O_{3}}(\mathbf{x}) \widetilde{O_{3}}(0)\right\rangle \sim 1 /|\mathbf{x}|^{1+\eta_{A}}$, but in principle it could be measured with a significantly extended analysis, if the transition is really of second order [ $\eta_{A}$ would show up in the functional form of Eq. (17)]. If the transition were of second order in the usual sense, then one could also measure the scalar correlation length critical exponents $\nu, \nu^{\prime}$ in the two phases, $m_{H} \sim\left|y-y_{c}\right|^{\nu, \nu^{\prime}}$, and the corresponding anomalous dimension at $y_{c}$. However, as we have not seen any critical behavior for the scalar correlation length, these exponents cannot be systematically measured. It seems that away from the critical point, $m_{H}$ shows approximate scaling where $\nu, \nu^{\prime}$ are consistent with $\frac{1}{2}$, see Fig. 5.

A more conclusive study of the infinite volume and continuum limits in the type-II regime would clearly be needed. Unfortunately this limit is numerically very demanding and requires much more extensive further simulations. Based on the present investigation, we can nevertheless point out that it would probably be more economic to use the noncompact lattice action than the compact one we have used here. The reason is that one can then take a large lattice spacing (smaller $\beta_{G}$ ) in order to get a larger physical volume, without having to worry about the nonzero value of the Polyakov mass.

As a final observation, let us note that one might expect the study of a gauge theory with a U(1) gauge group to be simpler than that with a more complicated group such as SU(2) (Refs. 9,10) or SU(2) $\times U(1) .{ }^{23}$ In fact, this turns out not to be the case: $\mathrm{U}(1)$ is numerically more demanding and requires larger lattices (at least in the compact formulation). The existence of a massless photon is not as such the only reason: there is a photon also in $\mathrm{SU}(2) \times \mathrm{U}(1)$, but there it exists in both phases and is not an order parameter.

\section{ACKNOWLEDGMENTS}

The simulations, totaling $4 \times 10^{15}$ flop $=130$ Mflops year, were carried out with a Cray C94 at the Finnish Center for Scientific Computing. We acknowledge useful discussions with B. Bergerhoff, J. Jersák, S. Khlebnikov, C. Michael, O. Philipsen, K. Rummukainen, M. Shaposhnikov, M. Tsypin, and G. Volovik.
*Electronic address: keijo.kajantie@cern.ch

† Electronic address: mikarjal@phcu.helsinki.fi

$\ddagger$ Electronic address: m.laine@ thphys.uni-heidelberg.de

$\S$ Electronic address: peisa@amtp.liv.ac.uk

${ }^{1}$ P.G. de Gennes, Solid State Commun. 10, 753 (1972); B.I. Halperin and T.C. Lubensky, ibid. 14, 997 (1974).

${ }^{2}$ B.I. Halperin, T.C. Lubensky, and S.-K. Ma, Phys. Rev. Lett. 32, 292 (1974).

${ }^{3}$ C. Dasgupta and B.I. Halperin, Phys. Rev. Lett. 47, 1556 (1981).

${ }^{4}$ J. Bartholomew, Phys. Rev. B 28, 5378 (1983).

${ }^{5}$ H. Kleinert, Gauge Fields in Condensed Matter (World Scientific, Singapore, 1989).
${ }^{6}$ H. Kleinert, Phys. Lett. 93A, 86 (1982); Lett. Nuovo Cimento 35, 405 (1982); Y. Munehisa, Phys. Lett. 155B, 159 (1985); K. Farakos, G. Koutsoumbas, and S. Sarantakos, Z. Phys. C 40, 465 (1988); A. Kovner, B. Rosenstein, and D. Eliezer, Nucl. Phys. B 350, 325 (1991); A. Kovner, P. Kurzepa, and B. Rosenstein, Mod. Phys. Lett. A 14, 1343 (1993); J. March-Russell, Phys. Lett. B 296, 364 (1992); W. Buchmüller and O. Philipsen, ibid. 354, 403 (1995); B. Bergerhoff, F. Freire, D.F. Litim, S. Lola, and C. Wetterich, Phys. Rev. B 53, 5734 (1996).

${ }^{7}$ M. Kiometzis, H. Kleinert, and A.M.J. Schakel, Phys. Rev. Lett. 73, 1975 (1994); I. Herbut and Z. Tešanović, ibid. 76, 4588 (1996); H. Kleinert and A.M.J. Schakel, supr-con/9606001 (un- 
published); cond-mat/9702159(unpublished); I.F. Herbut, J. Phys. A 30, 423 (1997); cond-mat/9702167(unpublished).

${ }^{8}$ It is interesting to note that in some related theories, namely an SU(2) gauge theory with a scalar field in the fundamental (Ref. 9) or adjoint (Ref. 10) representation, there is no first- or secondorder transition at $x>x_{c}$.

${ }^{9}$ K. Kajantie, M. Laine, K. Rummukainen, and M. Shaposhnikov, Phys. Rev. Lett. 77, 2887 (1996).

${ }^{10}$ A. Hart, O. Philipsen, J.D. Stack, and M. Teper, Phys. Lett. B 396, 217 (1997).

${ }^{11}$ G. Parisi, Statistical Field Theory (Addison-Wesley, Reading, MA, 1988), Chap. 5.

${ }^{12}$ K. Farakos, K. Kajantie, K. Rummukainen, and M. Shaposhnikov, Nucl. Phys. B 425, 67 (1994).

${ }^{13}$ P. Dimopoulos, K. Farakos, and G. Koutsoumbas, hep-lat/9703004; Z. Phys. C (to be published).

${ }^{14}$ M. Laine, Nucl. Phys. B 451, 484 (1995).

${ }^{15}$ If the plaquette part multiplied by $\beta_{G}$ were replaced with the noncompact version, then the coefficient 25.5 should be replaced with -1.1 .
${ }^{16}$ A. Polyakov, Phys. Lett. 59B, 82 (1975); Gauge Fields and Strings (Harwood, Chur, Switzerland, 1987).

${ }^{17}$ J. Ambjorn, A.J.G. Hey, and S. Otto, Nucl. Phys. B 210, 347 (1982).

${ }^{18}$ M.N. Barber, in Phase Transitions and Critical Phenomena, edited by C. Domb and J.L. Lebowitz (Academic, New York, 1983), Vol. 8.

${ }^{19}$ B. Berg and C. Panagiotakopoulos, Phys. Rev. Lett. 52, 94 (1984); H.G. Evertz, K. Jansen, J. Jersák, C.B. Lang, and T. Neuhaus, Nucl. Phys. B 285, 590 (1987).

${ }^{20}$ Indeed, the scalar mass has come down quite a lot since our first measurements (Ref. 21). This is because we have now a much better projection on the ground state and larger physical volumes.

${ }^{21}$ M. Karjalainen, M. Laine, and J. Peisa, Proceedings of Lattice 96 [Nucl. Phys. B (Proc. Suppl.) 53, 475 (1997)].

${ }^{22}$ O. Philipsen, M. Teper, and H. Wittig, Nucl. Phys. B 469, 445 (1996).

${ }^{23}$ K. Kajantie, M. Laine, K. Rummukainen, and M. Shaposhnikov, Nucl. Phys. B 493, 413 (1997). 